\title{
Influencia de la Separación de Residuos Sólidos Urbanos para Reciclaje en el Proceso de Incineración con Generación de Energía
}

\author{
José A. Poletto y Celso L. da Silva \\ Universidad Estadual Paulista, Departamento de Ingeniería Mecánica, Av. Luiz Edmundo \\ C. Coube, 14-01, Bauru, 17033-360 São Paulo, SP - Brasil \\ (e-mail: jpoletto@uol.com.br, celsos@feb.unesp.br)
}

\section{Resumen}

En este trabajo se analiza la recuperación de la energía de residuos sólidos urbanos (RSU) a través del proceso de incineración. Se tiene en cuenta la tendencia actual de separación de plásticos, papel y cartón, vidrio y metales, y su influencia en los flujos másicos y de energía en el sistema de la incineración de RSU. Para su desarrollo se utilizó la información relacionada con la generación de RSU de la ciudad de Bauru en Brasil, junto con el software COMBUST. Los resultados obtenidos permitieron estimar el poder calorífico teórico de los RSU típicos y también cuando se considera los residuos de papel, cartón y plásticos.

\section{Influence of the Separation of Municipal Solid Wastes to Recycling in the Incineration Process with Energy Generation}

\begin{abstract}
This work analyses the recuperation of the energy of Municipal Solid Waste (MSW) through the incineration process. It considers the up to date tendency of segregation (separation) of plastic, paper and cardboard, glass and metals and their influence in the fluxes of mass and energy in the incineration system of MSW. For its development was used information related to the generation of MSW in Bauru city and the Combust software. The results so obtained allowed the estimation of the Caloric power of the typical MSW and also of this residue when one considers the separations of paper/cardboard and plastic for recycling.
\end{abstract}

Keywords: solid wastes, incineration, energy generation, RSU, calorific power 


\section{INTRODUCCIÓN}

La recolección, tratamiento y disposición final de los Residuos Sólidos Urbanos (RSU) todavía es un problema en la mayoría de las ciudades de Brasil (Luiz, 2008) Los problemas de higiene, contaminación de las aguas subterráneas por las basuras, que implican residuos biológicos, es una preocupación constante cuando la disposición final del RSU es realizada en vertederos no controlados. La quema controlada de los RSU cuando se realiza en instalaciones con dispositivos adecuados de control de la contaminación del aire, permite una solución definitiva al problema, con bajo impacto ambiental, utilizando criterios técnicos adecuados.

Según Luiz (2008), Brasil tiene un gran potencial para generar energía a partir de los residuos sólidos y esta alternativa podría aumentar el suministro de energía en el país a 50 millones de megavatios-hora por año, lo que representa más del 15\% de la totalidad disponibles en la actualidad o alrededor de una cuarta parte de lo que genera la central hidroeléctrica de Itaipú. Hauser (2008), sostiene que la reutilización de la energía contribuirá a la solución de los problemas de gestión de los residuos sólidos en Brasil y en la reducción de las emisiones de gases de efecto invernadero para la protección del clima.

La composición del RSU es influenciada por varios factores: número de habitantes, poder adquisitivo, nivel educacional, hábitos y costumbres de la población, condiciones climáticas y estaciónales, y cambios en la política económica de un país. La composición física de los RSU de algunas ciudades paulistas, con características semejantes a las de Bauru, se muestran en la Tabla 1. En la Figura 1 se muestra la composición media del RSU en Brasil (IPT, 2001).

Tabla 1: Composición física del RSU (\%)

\begin{tabular}{|c|c|c|c|c|c|c|c|}
\hline Ciudad & Año & $\begin{array}{c}\text { Materia } \\
\text { Orgánica }\end{array}$ & $\begin{array}{c}\text { Papel y } \\
\text { cartón }\end{array}$ & Plástico & Vidrio & Metal & Otros \\
\hline Botucatu & 1997 & 74,11 & 7,61 & 8,41 & 1,99 & 3,86 & 4,02 \\
\hline São Carlos & 1989 & 56,7 & 21,3 & 8,5 & 1,4 & 5,4 & 6,7 \\
\hline Rio Claro & 1985 & 62,8 & 15,2 & 5,5 & 2,1 & 3,5 & 10,9 \\
\hline São Paulo & 1993 & 37,8 & 29,6 & 9,0 & 4,9 & 5,4 & 13,3 \\
\hline
\end{tabular}

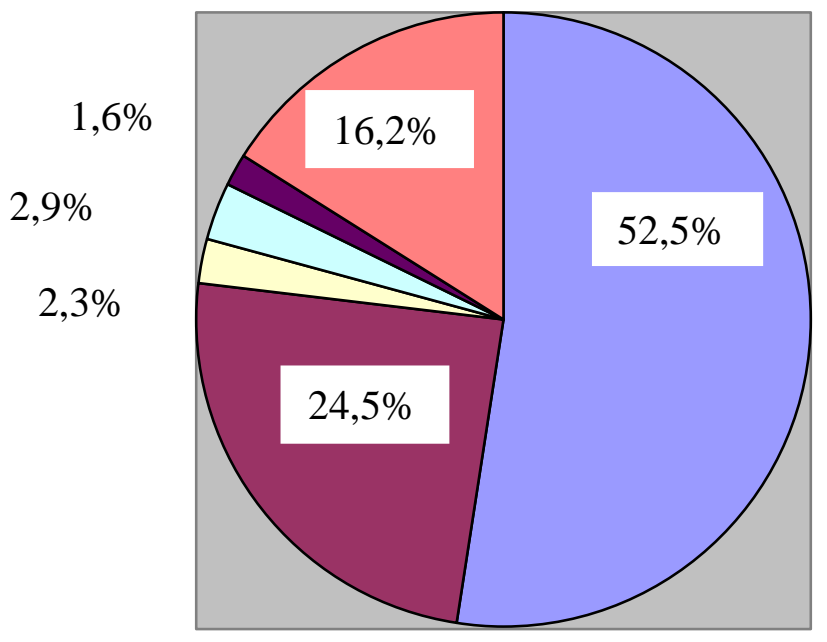

\begin{tabular}{|l|}
\hline Materia Orgánica \\
$\square$ Papel y cartón \\
$\square$ Metal \\
$\square$ Plástico \\
$\square$ Vidro \\
$\square$ Otros \\
\hline
\end{tabular}

Fig. 1: Composición media del RSU en Brasil

El reciclaje energético tiene estrecha relación con la incineración de RSU. Ya que la realizada en una instalación de combustión de RSU, difiere de la fábrica de incineración porque genera un producto, la energía (electricidad), que puede ser vendida, resultando en ahorro para el municipio. 
Según datos de la Agencia de Protección Ambiental de EUA (USEPA, 1989) cuando la incineración de los RSU tiene como objetivo la recuperación de energía, se debe conocer la composición de los RSU, que están directamente relacionadas a la cantidad de energía a ser recuperada. La humedad y la reducción de porcentaje de plásticos, por ejemplo, disminuyen la energía generada, elevando el costo de la tonelada incinerada y consecuentemente de la energía vendida. De acuerdo con la USEPA (1989), en Estados Unidos se estima que 75\% (en peso) de los RSU son combustibles; y en Brasil, la parte combustible de los RSU, varía en torno del 40\%. Con la actual tendencia de la segregación de materiales que tiene algún valor de mercado, encontrados en el RSU, se debe considerar la variación del Poder Calorífico en función del tipo y calidad del material segregado. El reciclaje es un conjunto de técnicas que tiene por finalidad aprovechar los residuos y utilizarlos nuevamente en el ciclo de producción del que salieron. Es el resultado de una serie de actividades por las cuales materiales que se volverían basura, son desviados, colectados, separados y procesados para ser usados como materia prima en la manufactura de nuevos productos. Este procedimiento puede influenciar el rendimiento de una usina de recuperación de energía, por ejemplo, la reducción en la cantidad de plásticos existentes en el RSU puede elevar el costo de la producción de energía, pues disminuye su poder calorífico. Las características de algunos materiales reciclables pueden ser resumidas en la Tabla 2.

Tabla 2: Características de algunos materiales reciclables

\begin{tabular}{|c|c|c|c|c|}
\hline Material & $\begin{array}{c}\text { Peso en el } \\
\text { RSU (\%) }\end{array}$ & $\begin{array}{c}\text { Reciclaje en } \\
\text { Brasil (\%) }\end{array}$ & $\begin{array}{c}\text { Precio Medio } \\
\text { de Mercado } \\
\text { (R\$/ton.) }\end{array}$ & $\begin{array}{c}\text { Poder } \\
\text { Calorífico } \\
\text { (kcal/kg) }\end{array}$ \\
\hline Papel flameado & 4,1 & 60 & 450,00 & 1.775 \\
\hline Papel y Cartón & 24 & 37 & 400,00 & 1.814 \\
\hline PEBD & 5 a 10 & 15 & 500,00 & 4.712 \\
\hline Plástico Rígido & 15 & 15 & 950,00 & 4.712 \\
\hline PET & 1,4 & 33 & 450,00 & 5.039 \\
\hline
\end{tabular}

En todo el mundo, aproximadamente 130 millones de toneladas de RSU al año se incineran en plantas de WTE (Waste to Energy) que producen unos 45 millones de kilovatios-hora de electricidad (Themelis, 2006).

La recuperación energética por la combustión del RSU, debe considerar los aspectos técnicos en la selección de los materiales de reciclado, además que papel e plásticos mejoran mucho el poder calorífico. Por ejemplo, una tonelada de viruta de papel flameado puede evitar el recorte de 10 a 12 árboles provenientes de plantaciones comerciales reforestadas. La fabricación de papel con uso de virutas gasta 10 a 50 veces menos agua que en el proceso tradicional que usa celulosa virgen, esto es reducir el consumo de energía a la mitad. La separación o no del Polietileno de Baja Densidad (PEBD) que junto con el Polietileno Tereftalato (PET) poseen alto poder calorífico, pero que liberan gases nocivos al medio ambiente cuando son incinerados. La generación de ésos gases exige sistemas de tratamiento, más sofisticados y eficientes, de descontaminación que implican en la generación de energía de más alto costo.

La determinación de la cantidad de RSU producido en una ciudad y sus diversos componentes no es simple, depende de una serie de factores, que mantienen una relación directa con el número de habitantes. De acuerdo con los datos del Instituto Brasileño de Geografía y Estadística (IBGE, 2000), la generación de RSU puede ser estimada de acuerdo a la población. En la Tabla 3 se encuentra la producción de RSU por persona basado en el número de habitantes de una determinada región brasileña.

Bauru es una típica ciudad de tamaño medio del interior paulista, contando hoy con aproximadamente 356.680 habitantes (IBGE, 2001). Genera diariamente $210.000 \mathrm{~kg}$ de RSU (SMA, 2007), que son destinados para el vertedero sanitario. Considerando los criterios de la Tabla 4 , Bauru genera 0,6 kg/hab.día de RSU, o sea, aproximadamente $214.000 \mathrm{~kg}$ por día. La estimación de las cantidades por habitante y totales de RSU generados, como su composición, puede ser 
divergente, pues depende de varios factores, tales como: época del año, tipo de colecta y pesaje, climatología, entre otros (Tchobanoglous y Theisen, 1996). El valor medio del Poder Calorífico Inferior $(\mathrm{PCl})$ de algunos materiales encontrados en el RSU está reunido en la Tabla 4 (Conesa, 2005).

Tabla 3: Índice de producción "per capita" de RSU en función de la población

\begin{tabular}{|c|c|}
\hline Población (hab.) & $\begin{array}{c}\text { Producción de RSU } \\
\text { (kg/hab.día) }\end{array}$ \\
\hline Hasta 100.000 & 0,4 \\
\hline De 100.001 a 200.000 & 0,5 \\
\hline De 200.001 a 500.000 & 0,6 \\
\hline Más que 500.001 & 0,7 \\
\hline
\end{tabular}

Tabla 4: Valor medio PCI de algunos componentes del RSU

\begin{tabular}{|c|c|}
\hline Material & PCI (kcal/kg) \\
\hline Residuo de comida & 700 \\
\hline Papel / cartón & 2.500 \\
\hline Textil & 3.400 \\
\hline Madera & 4.300 \\
\hline Plástico & 5.000 \\
\hline
\end{tabular}

El análisis elemental del RSU implica la determinación del porcentaje de Carbono (C), Hidrógeno $(\mathrm{H})$, Oxígeno (O), Nitrógeno (N), Azufre (S), y ceniza. Tal análisis es importante debido tanto al aspecto energético, como la preocupación con la emisión de compuestos clorados durante la incineración. En la Tabla 5 se encuentra una composición media, obtenida con datos americanos, pero que son utilizados en la mayoría de los trabajos sobre el asunto (Tchobanoglous y Theisen, 1996).

Tabla 5: Composición de los RSU Domésticos- Orgánicos

\begin{tabular}{|l|c|c|c|c|c|c|}
\hline & \multicolumn{7}{|c|}{ Porcentaje en masa (\% - Base seca) } \\
\hline Componentes & Carbono & Hidrógeno & Oxígeno & Nitrógeno & Azufre & Cenizas \\
\hline $\begin{array}{l}\text { Res.de } \\
\text { Comida }\end{array}$ & 48,0 & 6,4 & 37,6 & 2,6 & 0,4 & 5,0 \\
\hline Papel / cartón & 43,5 & 6,0 & 44,0 & 0,3 & 0,2 & 6,0 \\
\hline Plásticos & 60,0 & 7,2 & 22,8 & & & 10,0 \\
\hline Textiles & 55,0 & 6,6 & 31,2 & 4,6 & 0,15 & 2,5 \\
\hline Goma & 78,0 & 10,0 & & 2,0 & & 10,0 \\
\hline Cuero & 60,0 & 8,0 & 11,6 & 10,0 & 0,4 & 10,0 \\
\hline Jardín & 47,8 & 6,0 & 38,0 & 3,4 & 0,3 & 4,5 \\
\hline Madera & 49,5 & 6,0 & 42,7 & 0,2 & 0,1 & 1,5 \\
\hline
\end{tabular}

Según Tchobanoglous y Theisen (1996), en la Tabla 6 se encuentra la composición de la parte inorgánica que no irá a contribuir para el Poder Calorífico del RSU.

Según Silva y Velo (1997), la composición típica aproximada a los RSU depositados en el vertedero sanitario de Bauru aparece en la Tabla 7. Se observa en la Tabla 7, que la mayor parte del RSU esta constituida por el residuo de comida (55\%) y papel / cartón (21\%). El plástico es otro componente importante, pues, además de contribuir con 8,9\% en masa también tiene un Poder Calorífico elevado. Para la finalidad del presente artículo no se considera el porcentaje de vidrios, metales y otros, pues éstos son inertes y no influyen en la estimación del Poder Calorífico del RSU. Esta hipótesis está incluida en la columna Masa Seca Normalizada, donde los porcentuales de Masa Seca son ponderados dejando de considerarse vidrios, metales y otros. 
Tabla 6: Composición de los RSU Domésticos- Inorgánicos

\begin{tabular}{|l|c|c|c|c|c|c|}
\hline & \multicolumn{6}{|c|}{ Porcentaje en masa (\% - Base seca) - Inorgánicos } \\
\hline Componentes & Carbono & Hidrógeno & Oxígeno & Nitrógeno & Azufre & Cinzas \\
\hline Vidrios & 0,5 & 0,1 & 0,4 & $<0,1$ & & 98,9 \\
\hline Metales & 4,5 & 0,6 & 4,3 & $<0,1$ & & 90,5 \\
\hline Cenizas, etc & 26,3 & 3,0 & 2,0 & 0,5 & 0,2 & 68,0 \\
\hline
\end{tabular}

Tabla 7: Composición Típica de los Residuos del Vertedero Sanitario de Bauru, modificada.

\begin{tabular}{|l|c|c|c|c|}
\hline Componente & Masa Húmeda (\%) & $\begin{array}{c}\text { Humedad } \\
\text { (\%) }\end{array}$ & Masa Seca (\%) & $\begin{array}{c}\text { Masa seca } \\
\text { Normalizada (\%) }\end{array}$ \\
\hline $\begin{array}{l}\text { Res. de } \\
\text { Comida }\end{array}$ & 55,0 & 70,0 & 16,50 & 32,77 \\
\hline Papel y Cartón & 21,0 & 6,0 & 19,74 & 39,21 \\
\hline Textil y Cuero & 5,0 & 10,0 & 4,50 & 8,94 \\
\hline Madera & 1,1 & 20,0 & 0,88 & 1,75 \\
\hline Plástico & 8,9 & 2,0 & 8,72 & 17,33 \\
\hline Vidrio & 2,6 & 2,0 & 2,55 & - \\
\hline Metales & 5,4 & 2,0 & 5,29 & - \\
\hline Otros & 1,0 & 5,0 & 0,95 & - \\
\hline Total & 100 & & 59,13 & 100 \\
\hline
\end{tabular}

El presente trabajo tiene por objetivo principal estimar el Poder Calorífico Inferior de los RSU generados en el municipio de Bauru, tomándose en consideración la segregación de papeles / cartón y plásticos. Obtenido este parámetro, es de interés realizar una estimación teórica aproximada a la cantidad de energía que podría ser obtenida diariamente en la combustión de los RSU del municipio de Bauru.

\section{MATERIALES Y MÉTODOS}

Para la realización de este trabajo fueron utilizadas las informaciones disponibles en la literatura (Silva y Velo, 1997; Tchobanoglous y Theisen, 1996; Conesa, 2005).

Los cálculos fueron realizados utilizando el software COMBUST (Barreras y Pujol, 1996), que permite estimar los parámetros característicos del balance de masa de la combustión completa de combustibles sólidos, líquidos o gaseosos, tales como:

- El exceso de aire de combustión a partir de la concentración de $\mathrm{O}_{2}$ ( $\mathrm{o} \mathrm{CO}_{2}$ ) en los gases de combustión.

- Concentración máxima de $\mathrm{CO}_{2}$ en los gases de combustión.

- La temperatura de los gases de combustión en función del exceso de aire de combustión.

- La composición de masa y volumen de los gases de combustión, como entalpía, calor específico, densidad y masa molecular. Tanto en base seca como en base húmeda.

- El flujo de masa, o volumen, de los gases de combustión, secos o húmedos.

- El poder calorífico, densidad, masa molecular, calor específico y índice de Wobbe de hidrocarburos y sus mezclas. Tanto para tenor volumétrico como para el de masa y tanto para base húmeda como para base seca.

El software fue utilizado para la simulación de la combustión de diversas composiciones del RSU, considerando diferentes porcentajes de segregación de sus componentes, principalmente papel / cartón y plásticos. 


\section{RESULTADOS Y DISCUSIÓN}

A partir de los datos de las Tablas 5 y 7 , se genera la Tabla 8, que muestra el porcentaje de Carbono $(\mathrm{C})$, Hidrógeno $(\mathrm{H})$, Oxígeno $(\mathrm{O})$, Nitrógeno $(\mathrm{N})$, Azufre $(\mathrm{S})$, y ceniza en la base húmeda para cada componente del RSU

Tabla 8: Porcentaje de cada elemento de los componentes del RSU - base húmeda

\begin{tabular}{|l|c|c|c|c|c|c|}
\hline \multicolumn{7}{|c|}{ \% en masa - Base Húmeda } \\
\hline Componente & C & H & O & N & S & Ceniza \\
\hline Res. Comida & 14,4 & 1,9 & 11,3 & 0,8 & 0,1 & 1,5 \\
\hline Papel & 41,4 & 5,5 & 41,9 & 0,3 & 0,2 & 4,7 \\
\hline Plástico & 58,8 & 7,1 & 22,3 & & & 9,8 \\
\hline Textiles / cuero & 49,5 & 5,9 & 28,1 & 4,1 & 0,1 & 2,2 \\
\hline Goma & 76,4 & 9,8 & & 2 & & 9,8 \\
\hline Madera & 38,2 & 4,8 & 30,4 & 2,7 & 0,2 & 3,6 \\
\hline
\end{tabular}

Para la cantidad de RSU generado en el municipio de Bauru, se consideró la información de la Secretaría del Medio Ambiente de Bauru, esto es, $210.000 \mathrm{~kg} /$ día. Los datos de la Tabla 8 fueron utilizadas en el software COMBUST para determinación del PCl en el RSU.

En la Tabla 9 (Poletto, 2008) tiene si la contribución de cada componente del RSU para el PCl de un kilogramo de RSU, no considerando la segregación de materiales combustibles.

Tabla 9: Contribución de cada componente sin considerar la segregación

\begin{tabular}{|l|c|}
\hline Componente & Contribución \\
\hline Res. de Comida & 738 \\
\hline Papel y Cartón & 758 \\
\hline Textil y Cuero & 237 \\
\hline Madera & 38 \\
\hline Plástico & 521 \\
\hline Total PCI (kcal/kg de RSU) & $\mathbf{2 . 2 9 2}$ \\
\hline
\end{tabular}

Cuando se considera la segregación de papel / cartón y plásticos, el PCl disminuye según de acuerdo a lo mostrado en la Tabla 10.

Tabla 10: Contribución de cada componente del RSU, considerando la segregación total de papel / cartón y plástico.

\begin{tabular}{|l|c|}
\hline Componente & Contribución \\
\hline Res. de Comida & 738 \\
\hline Papel y Cartón & 0 \\
\hline Textil y Cuero & 237 \\
\hline Madera & 38 \\
\hline Plástico & 0 \\
\hline Total PCl (kcal/kg de RSU) & $\mathbf{1 . 0 1 3}$ \\
\hline
\end{tabular}

Se percibe que la práctica de la segregación en el origen de materiales que son buenos combustibles disminuye considerablemente el PCI del RSU. Así, se puede inferir que el Poder Calorífico Inferior del RSU de la ciudad de Bauru es aproximadamente $2.292 \mathrm{kcal} / \mathrm{kg}$, sin considerar la segregación y $1.013 \mathrm{kcal} / \mathrm{kg}$ en la hipótesis de segregación total de plásticos y papeles. 
Considerándose la segregación, en el origen, a través de fábricas de separación o por la acción de juntadores, principalmente de papel / cartón y plásticos se genera la Figura 2, donde puede ser visualizada la variación del PCI en función del porcentaje de papel / cartón y plástico segregado.

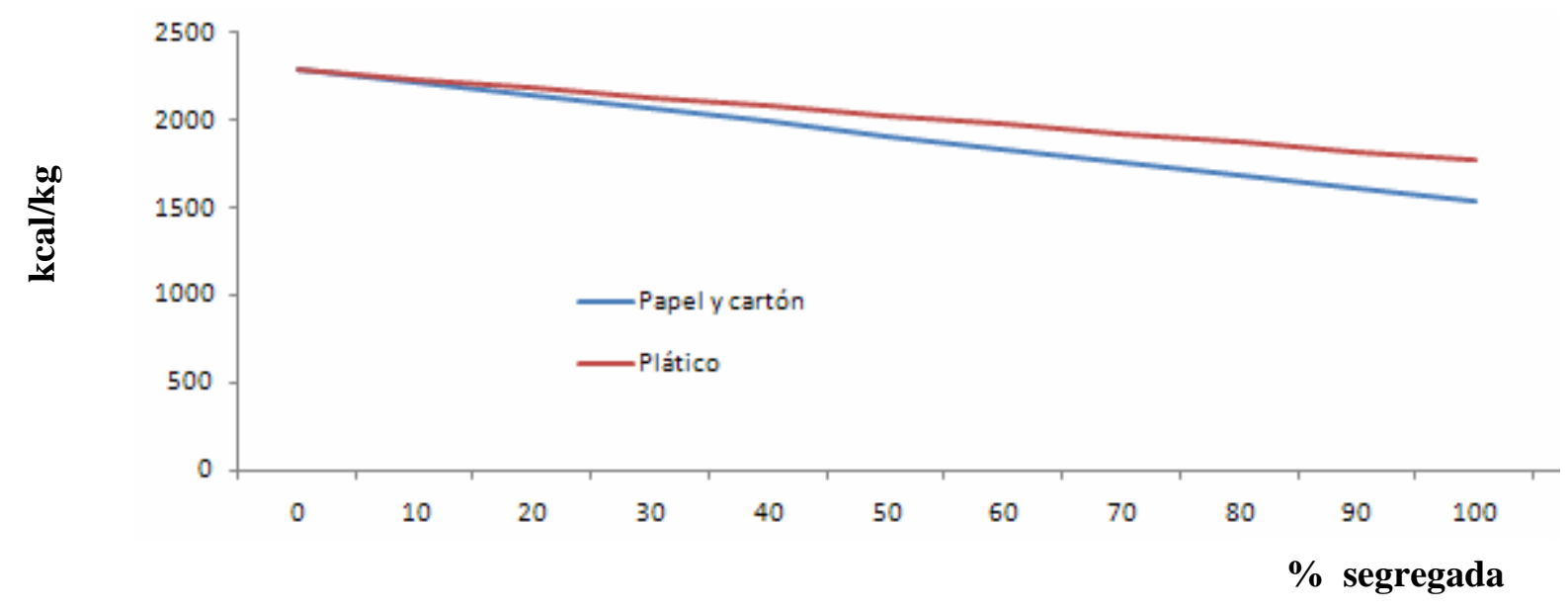

Fig. 2: Variación del PCl con la segregación de plástico y papel / cartón.

Como se esperaba, la reducción en las cantidades de materiales que son buenos combustibles, tales como papel / cartón y plástico, reducen el Poder Calorífico del RSU, implicando en menores cantidades de energía que pueden ser recuperadas (Poletto y Silva, 2008).

\section{CONCLUSIONES}

La adopción de un sistema de separación y segregación en el origen de materiales con valor de mercado, principalmente de aquéllos que son mejores combustibles, por cierto contribuirá a la reducción de la cantidad de energía que puede ser recuperada a través del proceso de incineración controlada.

El Poder Calorífico del residuo sólido urbano es gradualmente reducido en la medida en que se reduce previamente las cantidades de papel / cartón y plásticos, proporcionando temperaturas de combustión y eficiencia del proceso de combustión menores.

Teóricamente y considerando una eficiencia del 28\% para una planta de incineración de RSU, los residuos del municipio de Bauru podrían generar aproximadamente 156.408 kWh por día sin considerar la segregación, y 117.819 kWh por día cuando es considerada la segregación.

\section{REFERENCIAS}

Barreras, M.A.L. y R.O. Punjol; La Combustión, Universidade de Engenharia Técnica de Barcelona, (pg. 45),Ed. Cenac, (1996).

Conesa, J.A.; Incineração de Resíduos Sólidos, Universidade de Alicante, Departamento de Engenharia Química, Espanha, Disponible en: < http://iq.ua.es/> (2005).

Hauser, P.D.; Incineração de Resíduos Sólidos Mostra Caminhos Para Desenvolvimento Sustentável, Universidade Federal do Rio de Janeiro, Disponible en: http://integracao. fgvsp.br/ano9/06/pesquisas.htm (2006).

IBGE - Fundação Instituto Brasileiro de Geografia e Estatística, Sinopse preliminar do censo demográfico, Disponible en: <http://www.ibge.gov.br/>,São Paulo, (2000).

IBGE - Fundação Instituto Brasileiro de Geografia e Estatística, Inventário Estadual de Resíduos Sólidos Domiciliares, Disponible en:<http://www.ibge.gov.br/>, São Paulo, (2001).

Información Tecnológica Vol. - 20 No 2 - 2009 
IPT - Instituto de Pesquisas Tecnológicas do Estado de São Paulo. Lixo Municipal: Manual de Gerenciamento Integrado. $2^{\circ}$ ed. São Paulo: IPT/CEMPRE (2001).

Luiz, J.A.M.; O lixo da energia e a energia do lixo: problemas e soluções, Disponible en $<$ http://www.ecoeacao.com.br/index2.php?option=com_content\&do_pdf=1\&id=6670>, acesso en março 2008 (2008)

Poletto, J.A.; Viabilidade Energética e Econômica da Incineração de Resíduo Sólido Urbano Considerando a Segregação para Reciclagem, dissertação de mestrado, Departamento de Engenharia Mecânica, UNESP, Bauru (2008).

Poletto, J.A. y C.L. Silva; Energy Aspects of the RSU Incineration. In: Symposium Brazil-Japan in Economy, Science and Technological Innovation, Disponible en: <http://www.athena.biblioteca. unesp.br/F/2UID2XSUXB4UH45P12NNN85AVACN5JHF2FYGNYI35QQT8VNEHE-3209?func=itemglobal\&doc library=UEP01\&doc number=000558121\&year=\&volume=\&sub library=BBA $>, \quad$ Sao Paulo (2008).

SMA, secretaria do Meio Ambiente de Bauru, Disponible en: http://www.bauru.sp.gov.br/prefeitura/ pmb.php?acao=cat\&action=ler\&cat=8\&news_id=122, (2007)

Silva, C.L. y E.G. Velo; "Incineração de Resíduos Sólidos de Serviços de Saúde Utilizando o Biogás de Aterros Sanitários", XIV Congresso Brasileiro de Engenharia Mecânica - COBEM 97, Bauru-SP, (1997).

Tchobanoglous, G. y H.S. Theisen; Integrated Solid Waste Management: Engineering Principles and Management Issues (pg. 669), McGraw-Hill, Inc. International Ed. (1996).

Themelis, N.J.; An overview of the global waste-to-energy industry, Disponible en: < www.iswa.org>, (2006)

USEPA - United States Environmental Protection Agency. Decision-makers guide to waste management (1989). 